\title{
Influence of turbulence on the dynamo threshold
}

\author{
J-P. Laval ${ }^{1}$, P. Blaineau ${ }^{2}$, N. Leprovost ${ }^{2}$, B. Dubrulle ${ }^{2}$ and F. Daviaud ${ }^{2}$ \\ 1 Laboratoire de Mécanique de Lille, CNRS, UMR 810\%, \\ Blv Paul Langevin, F-59655 Villeneuve d'Ascq Cedex, France \\ 2 SPEC/DRECAM/DSM/CEA Saclay and CNRS, \\ URA2464, F-91190 Gif sur Yvette Cedex, France
}

\begin{abstract}
We use direct and stochastic numerical simulations of the magnetohydrodynamic equations to explore the influence of turbulence on the dynamo threshold. In the spirit of the Kraichnan-Kazantsev model, we model the turbulence by a noise, with given amplitude, injection scale and correlation time. The addition of a stochastic noise to the mean velocity significantly alters the dynamo threshold. When the noise is at small (resp. large) scale, the dynamo threshold is decreased (resp. increased). For a large scale noise, a finite noise correlation time reinforces this effect.

PACS numbers: 47.27.Eq, 47.27.Sd, 47.65.+a, 91.25.Cw
\end{abstract}

The process of magnetic field generation through the movement of an electrically conducting medium is called a dynamo. When this medium is a fluid, the instability results from a competition between magnetic field amplification via stretching and folding, and damping through magnetic diffusion. This is quantified by the magnetic Reynolds number $R m$, which must exceed some critical value $R m_{c}$ for the instability to operate. Despite their obvious relevance in natural objects, such as stars, planets or galaxies, dynamos are not so easy to study or model. Computer resources limit the numerical study of dynamos to a range of either small Reynolds numbers Re (laminar dynamo), modest $R m$ and $R e$ [1] or small $P m=R m / R e$ using Large Eddy Simulation [2]. These difficulties explain the recent development of experiments involving liquid metals, as a way to study the dynamo problem at large Reynolds number. In this case, the flow has a non-zero mean component and is fully turbulent. There is, in general, no exact analytical or numerical predictions regarding the dynamo threshold. However, prediction for the mean flow action can be obtained in the so-called "kinematic regime" where the magnetic field back reaction onto the flow is neglected (see e.g. 3]). This approximation is very useful when conducting optimization of experiments, so as to get the lowest threshold for dynamo action based only on the mean flow $R m_{c}^{M F}$ [4, 5, 6, 7]. It led to very good estimate of the measured dynamo threshold in the case of experiments in constrained geometries [8], where the instantaneous velocity field is very close to its time-average.

In contrast, unconstrained experiments [7, [9] are characterized by large velocity fluctuations, allowing the exploration of the influence of turbulence onto the meanflow dynamo threshold. Theoretical predictions regarding this influence are scarce. Small velocity fluctuations produce little impact on the dynamo threshold [10]. Predictions for arbitrary fluctuation amplitudes can be reached by considering the turbulent dynamo as an instability (driven by the mean flow) in the presence of a multiplicative noise (turbulent fluctuations) 11]. In this context, fluctuations favor or impede the magnetic field growth depending on their intensity or correlation time. This observation is confirmed by recent numerical simulations of simple periodic flows with non-zero mean flow [12, 13] showing that turbulence increases the dynamo threshold.

In the sequel we use direct and stochastic numerical simulation of the magnetohydrodynamic (MHD) equations to explore a possible explanation, linked with the existence of non-stationarity of the largest scales. We found that the addition of a stochastic noise to the mean velocity could significantly alter the dynamo threshold. When the noise is at small scale, the dynamo threshold is decreased, while it is increased for a large scale noise. In the latter case, the noise correlation time plays a role, and reinforces this effect, as soon as it is larger than the mean eddy turnover time. When interpreted within the Kraichnan-Kazantsev model of MHD flow, these results predict that large scale (resp. small scale) turbulence inhibits (resp. favors) dynamo action.

The MHD equations for incompressible fluids are :

$$
\begin{aligned}
\partial_{t} \mathbf{u}+\mathbf{u} \cdot \nabla \mathbf{u} & =-\nabla P+\nu \nabla^{2} \mathbf{u}+\mathbf{j} \times \mathbf{B}+f(t) \mathbf{v}^{T G}, \\
\partial_{t} \mathbf{B} & =\nabla \times(\mathbf{u} \times \mathbf{B})+\eta \nabla^{2} \mathbf{B} .
\end{aligned}
$$

Here, $\mathbf{u}$ is the velocity, $\mathbf{B}$ is the Alfven velocity, $P$ the pressure, $\nu$ the viscosity, $\eta$ the magnetic diffusivity, $\mathbf{j}=$ $\nabla \times(\mathbf{B})$, and $\mathbf{v}^{T G}=(\sin x \cos y \cos z,-\cos x \sin y \cos z, 0)$ is the Taylor-Green vortex and $f(t)$ is set by the condition that the $(1,1,1)$ Fourier components of the velocity remains equal to $\mathbf{v}^{T G}$. The equations are integrated on a triply periodic cubic domain using a pseudo-spectral method. The aliasing is removed by setting the solution of the $1 / 3$ largest modes to zero. The time marching is done using a second-order finite difference scheme. An Adams-Bashforth scheme is used for the nonlinear terms while the dissipative terms are integrated exactly. The two control parameters are the Reynolds number $R e=$ $v_{r m s} l_{\text {int }} / \nu$ and the magnetic Reynolds number $R m=$ $v_{r m s} l_{\text {int }} / \eta$, where $v_{r m s}=(1 / 3) \sqrt{2 E}=(1 / 3) \sqrt{<u^{2}>}$ is the (spatial) r.m.s. velocity based on the total kinetic 
energy $E=\int E(k) d k$ and $l_{\text {int }}=(3 \pi / 4) E / \int k E(k) d k$ is the integral scale of the turbulent flow. Both $v_{r m s}$ and $l_{\text {int }}$ fluctuate with time. Thus, viscosity and diffusivity are dynamically monitored so as to keep $R e$ and $R m$ constant. We have checked that $R e$ is a simple linear function of a non-dynamical Reynolds number $R e_{\exp }=v_{\max } \pi / \nu$ (usually used in experiments) based on maximum velocity and half the simulation box: $R e=7.41 R e_{\text {exp }}$. In the sequel $\langle X\rangle($ resp. $\bar{X})$ refers to spatial (resp. time) average of $X$.

We ran typically four types of simulations : i) DNSMHD, where the full set of equation (11) is integrated at $5 \leq R e \leq 100$ and $5 \leq R m \leq 50$ using resolutions up to $256^{3}$; ii) LES-MHD, where the Lesieur-Chollet model of turbulence is used for the velocity equation (1T. a), allowing to explore a case out-of-reach of DNS 12], namely $R e=500,5 \leq R m \leq 100$; iii) kinematic simulations ; iv) kinematic-stochastic (KS) simulations. In these last two cases, only the induction equation (1)-b) is integrated with $\mathbf{u}$ set to a given velocity field. In the kinematic case, it corresponds to the stationary velocity field $\overline{\mathbf{u}}(R e)$ obtained through time-average of a stable solution of the Navier-Stokes equations with Taylor-Green forcing, at fixed Reynolds number. This procedure is complicated by the presence of hydrodynamic instabilities at low Reynolds number [14], which impose very long simulation time (typically over 1000 s, i.e. 400 eddy turn over times at $R e=46$ ) to ensure convergence towards an asymptotically stable solution. The average is then performed over several (typically 200) eddy-turnover times. In the KS case, the velocity field $\mathbf{u}=\overline{\mathbf{u}}(R e)+\mathbf{v}^{\prime}\left(k_{I}, \tau_{c}\right)$ is the sum of a time averaged velocity field at a given $R e$ and of an external Markovian Gaussian noise, with fixed amplitude $v^{\prime}$, correlation time $\tau_{c}$ and typical scale $k_{I}$. In both kinematic simulations, the magnetic Reynolds number $R m$ is computed by using the rms velocity and integral scale of $\mathbf{u}$. In the deterministic case, this amounts to use $V_{r m s}=(1 / 3) \sqrt{\left\langle\bar{u}^{2}\right\rangle}$ and $L_{i n t}$ the (spatial) rms velocity and integral scale of the time-averaged velocity field, therefore respecting the experimental procedure followed in optimization of dynamo experiments [4, 5, 6, 7].

For each type of simulation, we fix $\operatorname{Re}\left(v^{\prime}, \tau_{c}\right.$ and $k_{I}$, if needed), vary $R m$ and monitor the time behavior of the magnetic energy $<B^{2}>$ and the finite-time Lyapunov exponent $\Lambda=0.5 \partial_{t}<\ln \left(B^{2}\right)>$, where the average is taken over the spatial domain. Three types of behaviors are typically observed [14]: i) no dynamo : the magnetic energy decays, the Lyapunov converges towards a finite negative value; ii) undecided state : the magnetic energy remains at a low level, with intermittent bursts of magnetic energy [15] and oscillation of the Lyapunov, so that no fit of the Lyapunov exponent can be obtained; iii) turbulent dynamo : the magnetic energy grows with positive Lyapunov, and, in the DNS-MHD or LES-MHD, reaches a nonlinear saturated regime.

From the values of the Lyapunov in the turbulent dy- namo and no dynamo regime, one may derive the critical magnetic Reynolds number $R m_{c}(R e)$, solution of $\Lambda\left(R e, R m_{c}\right)=0$, through a standard interpolation procedure.

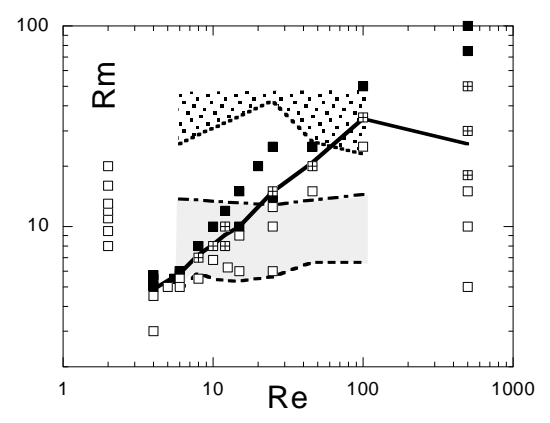

FIG. 1: Simulation parameter space. Square refer to DNSMHD and LES-MHD simulations, and shaded areas to windows of dynamo action for kinematic simulations with mean flow. $\square$ : no-dynamo case ; $\boxplus$ : undecided state; $\mathbf{\square}$ : dynamo case $;-R m_{c}^{\text {turb }} ;---R m_{c}^{M F} ;-\cdot-$. end of the first kinematic dynamo window ; $\cdots$ beginning of the second kinematic dynamo window. Shaded areas indicate explored windows of dynamo action for the mean flow.

A summary of our exploration of the parameter space is provided in Fig. 1, for the non-stochastic simulations, where the only control parameters are $R m$ and $R e$. We did not detect any dynamo at $R e=2$. Between $R e=4$ and $R e=6$, we observed heterocline dynamos, oscillating between a non-dynamo and a dynamo state. The window $2<R e<4$ has been studied in [12], where decreasing critical magnetic Reynolds number has been found. For $4<R e<100$, we found that the critical magnetic Reynolds number for dynamo action in a turbulent flow $R m_{c}^{\text {turb }}$ increases with the Reynolds number, in quantitative agreement with the result obtained in the same geometry, but with a different forcing (at constant force instead of constant velocity) [12]. Our LES-MHD simulation confirms the saturation of the dynamo threshold at large Reynolds number already observed in constant force simulations 12]. For the mean flow, we have actually detected at least two windows of dynamo actions : one, independent of $R e$, starting above $R m_{c}^{M F} \approx 6$ and centered around $R m=10$, with real Lyapunov (stationary dynamo); a second, occurring at larger $R m$, varying with $R e$, with complex Lyapunov (oscillatory dynamo). One sees that $R m_{c}^{\text {turb }}$ varies across these two windows and always exceeds $R m_{c}^{M F}$. In the sequel, we show that the increase and saturation of $R m_{c}^{\text {turb }}$ is not due to a crossing between the two dynamo modes, but to the influence of non-stationary large scales over $R m_{c}^{M F}$.

To make an easier connection between DNS and KS simulations, we introduce a parameter that quantifies the noise intensity, $\delta=\overline{\left\langle u^{2}\right\rangle} /\left\langle\bar{u}^{2}\right\rangle$. This parameter depends on the noise amplitude, as well as its correlation 

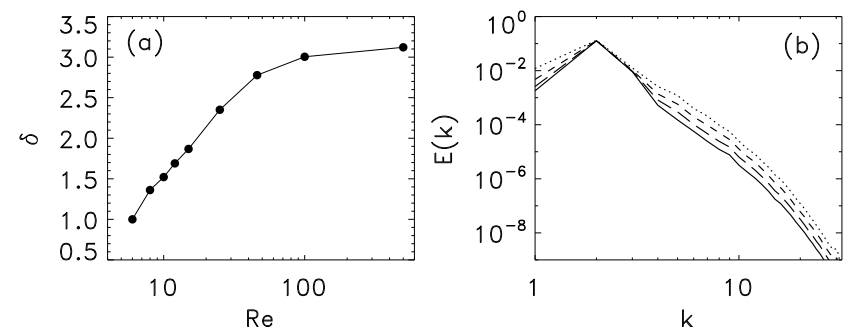

FIG. 2: a) Noise intensity $\left.\delta=\overline{\left\langle u^{2}>\right.} /<\bar{u}^{2}\right\rangle$, as a function of the Reynolds number, measured in our DNS simulations under the dynamo threshold. b) Energy spectrum of the velocity field in the DNS, at $R e=46$, for different average period $T$ : dotted line : $T=0$; short dashed line : $T=75 \mathrm{~s}$; long-dashed line : $T=150 \mathrm{~s}$; continuous line : $T=300 \mathrm{~s}$.

time and characteristic scale, and need to be computed for each stochastic simulation. It can also be computed in the direct simulations, and is found to depend onto the Reynolds number, increasing from a value of 1 at low Reynolds number, until about 3 at the largest available Reynolds number (Figure 2-a). Note that $\delta-1$ is just the ratio of the kinetic energy of fluctuations onto the kinetic energy of the mean flow. In the sequel, the comparison between the KS and DNS-MHD simulations will therefore be made using $\delta$ as the control parameter. Another interesting information can be obtained from the energy spectrum of the velocity field, as one averages over longer and longer time scales (Figure2-b). One sees that during the first period of average (typically, a few eddy turn-over time, i.e. about 5 to $10 \mathrm{~s}$ ), one mainly removes the fluctuations at largest scales, while the remaining average mostly removes small scales (over time scales of the order of 50 to 100 eddy-turnover times, i.e. $300 \mathrm{~s}$ ).

In the sequel, we explore the influence of both type of fluctuations through the KS simulations, by considering noise at large $\left(k_{I}=1\right)$ and small scale $\left(k_{I}=16\right)$, with correlation time ranging from 0 to $50 \mathrm{~s}$. Since the kinematic dynamo threshold is essentially constant for all values of the Reynolds number we explored, we first focus on the study of the case where the time-averaged field is fixed as $\bar{u}(R e=6)$ and vary the noise amplitude, characteristic scale or correlation time, to explore their influence on the dynamo threshold. An example of our exploration of the parameter space is provided in Figure 3 for different kinds of noise and $\bar{u}(R e=6)$. Note that by using our external noise, we are able to produce noise intensities of the order of noise intensities measured in experiments $\left(\delta \sim 10\right.$ at $R e \sim 10^{6}$ for the von Karman flow), and that are out of reach of DNS. For low correlation time or injection scale, we are actually able to follow the deformation of the two windows of dynamo action. One sees that a noise does not destroy them, but rather distorts them. In the case where the noise is at small scale $\left(k_{I}=16\right)$, the two windows are
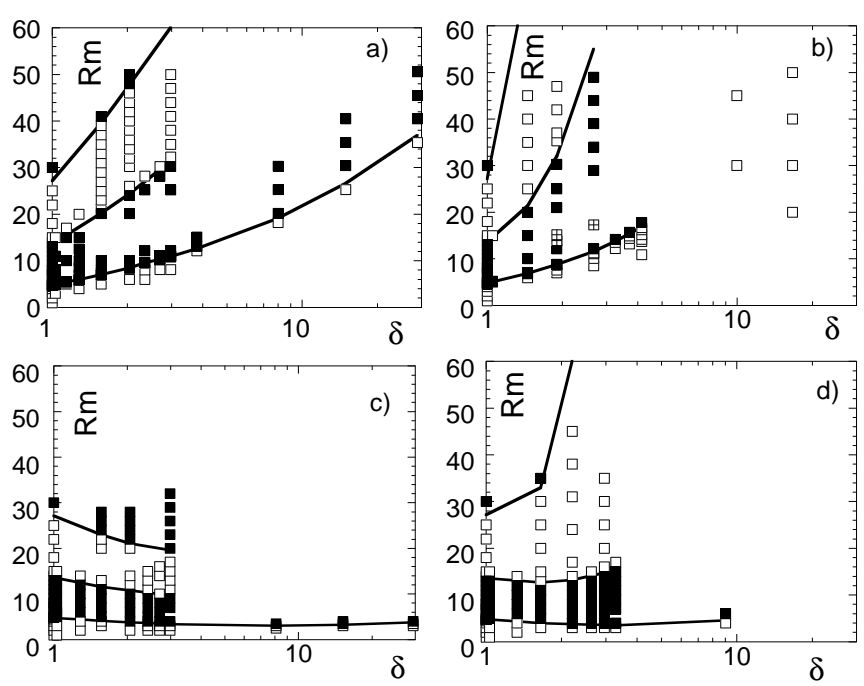

FIG. 3: Parameter space for noise at $R e=6$ for different noise parameters : a) $\tau_{c}=0, k_{I}=1$; b) $\tau_{c}=0.1 \mathrm{sec}, k_{I}=1$; c) $\tau_{c}=0, k_{I}=16$; d) $\tau_{c}=0.1 \mathrm{sec}, k_{I}=16$. $\square$ : no-dynamo case; $\boxplus$ : undecided state; $\mathbf{\square}$ : dynamo case. The full lines are zero-Lyapunov lines.

slightly tilted downwards, while they are lifted upwards in the case of large scale noise $\left(k_{I}=1\right)$. The influence of the noise onto the first dynamo bifurcation (the dynamo threshold) can be summarized by plotting the critical magnetic Reynolds numbers as a function of the noise intensity (Fig. 4. 4). Large scale (resp. small-scale) noise tends to increase (resp. decrease) the dynamo threshold. Furthermore, one sees that for small scale noise, the decrease in the dynamo threshold is almost independent of the noise correlation time $\tau_{c}$, while for the large scale noise, the increase is proportional to $\tau_{c}$ at small $\tau_{c}$. At $\tau_{c} \gtrsim 1$ sec-one third of the mean eddy-turnover time-, all curves $R m_{c}(\delta)$ collapse onto the same curve. We have further investigated this behavior to understand its origin. Increasing $\delta$ first increases of the flow turbulent viscosity $\overline{v_{r m s}} \overline{l_{i n t}}$ with respect to its mean flow value $V_{r m s} L_{\text {int }}$. This effect can be corrected by considering $R m_{c}^{*}=R m_{c} V_{r m s} L_{i n t} / \overline{v_{r m s}} \overline{l_{i n t}}$. Second, an increase of $\delta$ produces an increase of the fluctuations of kinetic en-

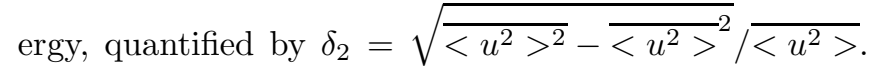
This last effect is more pronounced at $k_{I}=1$ than at $k_{I}=16$. It is amplified through increasing noise correlation time. We thus re-analyzed our data by plotting $R m_{c}^{*}$ as a function of $\delta_{2}$ (Fig. 4. $\mathrm{b}$ ). All results tend to collapse onto a single curve, independently of the noise injection scale and correlation time. This curve tends to a constant equal to $R m_{c}^{M F}$ at low $\delta_{2}$. This means that the magnetic diffusivity needed to achieved dynamo action in the mean flow is not affected by spatial velocity fluctuations. This is achieved for small scale noise, or large scale noise with small correlation time scale. In contrast, 
the curve diverges for $\delta_{2}$ of the order of 0.2 , meaning that time-fluctuations of the kinetic energy superseding 20 percent of the total energy annihilate the dynamo.

We now turn to detailed comparison of dynamo thresholds obtained in KS simulation with DNS-MHD case. In Fig. [5 we show that the dynamo threshold obtained at $k_{I}=1$, for $\tau_{c} \gtrsim 1$ as a function of the noise intensity corresponds to the DNS-MHD dynamo threshold. Note that the noise intensity $\delta$ saturates past a Reynolds number of about 100 (Fig. 2-a), thereby inducing the saturation of the critical magnetic Reynolds number at large Reynolds number. To check that our results are not affected by the choice of $\bar{u}$, we ran additional KS simulations with $\bar{u}$ computed at $R e=25,46$ and 100. Since the computational cost in these cases is much larger than in the case $R e=6$, we focused on the case where the noise has a correlation time $\tau_{c}=1$ or $8 \mathrm{sec}$. and injection scale $k_{I}=1$ and only computed the critical magnetic Reynolds number for the level of noise reached by the DNS at that Reynolds number. Figure 5 shows that the dynamo threshold coincides with the dynamo thresholds both of KS simulations at $R e=6$ and of the DNS, indicating that a large scale noise is probably responsible from the increase of $R m_{c}^{\text {turb }}$ with Reynolds number. A physical identification of the dynamics of the velocity fluctuations playing the role of this noise can be performed by visual inspection of the turbulent velocity field. One observes that the large scale vortices generated by the Taylor-Green forcing are not exactly stationary, but wander slightly with time. A similar large-scale non-stationarity has been observed in the shear layer of Von Karman flows 16, 17]. This process is approximately reproduced by a large scale noise with sufficiently long correlation time, and may therefore been held responsible for the significant increase of the dynamo threshold.

Our work suggests that it might not be so easy to achieve turbulent dynamos in unconstrained geometries, with large scale non-stationarity. In the experiments, a necessary ingredient for dynamo action could therefore be a monitoring of the large scale, so as to keep them as stationary as possible. In geo- and astrophysical flows, this role could be played by the Coriolis force. Our work also indicates that a well chosen noise can be used in place of the actual turbulent velocity fluctuations to compute the dynamo threshold, at a much lower computational cost. In some sense, a kinematic-stochastic simulation can therefore be seen as a turbulent model and might be useful in the astro- or geophysical context.

Acknowledgments We thank the GDR Turbulence and GDR Dynamo for support, J-F. Pinton, Y. Ponty, A. Chiffaudel and F. Plunian for discussions, and E. Gouillard for logistical help and CPU. Numerical simulations were performed at IDRIS.
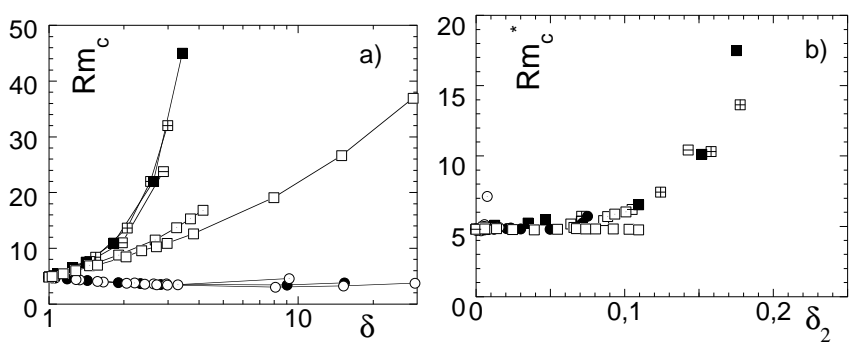

FIG. 4: Evolution of the dynamo threshold for KS simulations with $\bar{u}(R e=6)$. a) $R m_{c}$ as a function of $\delta$ and b) $R m_{c}^{*}$ as a function of $\delta_{2}$ for different noise parameters : $k=1: \square$ $\tau_{c}=0 ; \square \tau_{c}=0.1 \mathrm{sec} ; \boxminus \tau_{c}=1 \mathrm{sec} ; \boxplus \tau_{c}=8 \mathrm{sec} ; \mathbf{\square} \tau_{c}=50$ sec $; k=16: \circ \tau_{c}=0 ; \odot \tau_{c}=0.1 \mathrm{sec} ; \bullet \tau_{c}=50 \mathrm{sec}$.

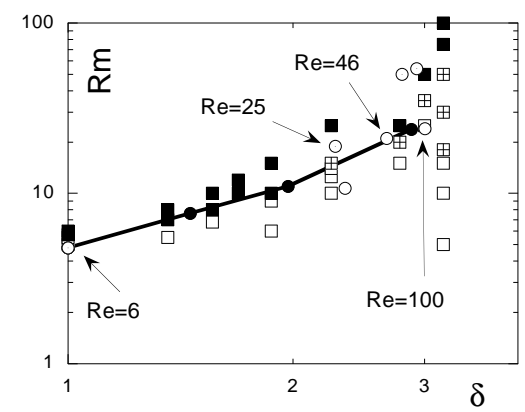

FIG. 5: Evolution of the dynamo threshold as a function of $\delta$ for the DNS (squares, same meaning as in Fig. 1 and 3) and KS simulations with $k_{I}=1$ using $\bar{u}(R e=6), \tau_{c}=1$ $\sec (\bullet$ connected with line), and $\bar{u}$ at the Reynolds number corresponding to the DNS with equivalent $\delta$ ( $\circ$ for $\tau_{c}=1 \mathrm{sec}$ and $\odot$ for $\tau_{c}=8 \mathrm{sec}$ ).

[1] G. A. Glatzmaier and P. H. Roberts, Nature 377, 203 (1995) ; C. Nore et al., Physics of Plasmas 41 (1997) ; A. Schekochihin et al., Phys. Rev. Lett. 92, 054502 (2004) ; S. Boldyrev and F. Cattaneo, Phys. Rev. Lett. 92, 144501 (2004).

[2] Y. Ponty et al., Phys. Rev. Letters 92, 144503, (2004) ;

[3] M. L. Dudley and R. W. James, Proc. R. Soc. London 425 (1989).

[4] F. Stefani et al., in Transfer Phenomena in Magnetohydrodynamic and Electroconducting Flows, Ph. Marty et al., eds, 31 (Kluwer) (1999).

[5] L. Marié et al., Eur. Phys. J. B 33, 469 (2003).

[6] F. Ravelet et al., Phys. Fluids 17, 117104 (2005).

[7] C.B. Forest et al., Magnetohydrodynamics 38, 107 (2002).

[8] A. Gailitis et al., Phys. Rev. Lett. 86, 3024 (2001); R. Stieglitz and U. Müller, Phys. Fluids 13, 561 (2001)

[9] N. L. Peffley and A. B. Cawthorne and D. P. Lathrop, Phys. Rev. E 61, 5 (2000); M. Bourgoin et al, Phys. Fluids 14, 3046 (2002).

[10] S. Fauve and F. Pétrélis. The dynamo effect, Peyresq Lectures on Nonlinear Phenomena, Vol. II, J.-A. Sepulchre 
ed., World Scientific, Singapore (2003).

[11] N. Leprovost and B. Dubrulle, Europhys. J. B 44, 395 (2005).

[12] Y. Ponty et al., Phys. Rev. Letters 94, 164502 (2005) ; Y. Ponty et al., submitted to Phys. Rev. Letters (2005).

[13] R.A. Bayliss, C.B. Forest and P. Terry, submitted to Phys. Rev. Letters (2004).
[14] J-P Laval et al., submitted to Europhys. J. B (2005).

[15] Such intermittent bursts have already been reported near the dynamo threshold in a modified ABC flow by D. Sweet et al., Phys. Plasmas, 8, 1944 (2001).

[16] F. Ravelet, PhD Thesis, Ecole Polytechnique (2005).

[17] R. Volk, PhD Thesis, ENS Lyon (2005). 